\title{
miR-331-3p suppresses cell invasion and migration in colorectal carcinoma by directly targeting NRP2
}

\author{
HONGYE ZHANG, RUIYU WANG and MINGXIA WANG \\ Department of Oncology, Linyi Central Hospital, Linyi, Shandong 276400, P.R. China
}

Received March 20, 2019; Accepted July 30, 2019

DOI: $10.3892 /$ ol.2019.11029

\begin{abstract}
Colorectal carcinoma (CRC) is a common tumor of the digestive system with poor prognosis. Studies have shown that aberrant microRNA (miRNA) expression can affect CRC progression by regulating target genes. In the present study, we investigated the functional roles and potential mechanisms of miR-331-3p in CRC. The expression of miR-331-3p and neuropilin-2 (NRP2) in CRC was detected by RT-qPCR. Then, Transwell assays were conducted to investigate the influence of miR-331-3p on CRC cell invasion and migration abilities. Luciferase reporter assays were performed to determine the target gene of miR-331-3p. It was found that miR-331-3p expression was notably declined in CRC and inversely correlated with the NRP2 expression. miR-331-3p upregulation significantly inhibited CRC cell invasion and migration. Additionally, western blot analysis demonstrated that miR-331-3p restoration evidently suppressed CRC cell EMT. Moreover, NRP2 was conformed to be a novel target of miR-331-3p and knockdown of NRP2 partially inversed the effects of the miR-331-3p inhibitor on cell invasion and migration. These results suggested that miR-331-3p exerted tumor suppressive roles in CRC by targeting NRP2 and miR-331-3p/NRP2 may serve as a potential therapy for CRC.
\end{abstract}

\section{Introduction}

Colorectal carcinoma (CRC) is a common digestive system carcinoma, remaining one of the major factors of tumor deaths globally (1). As there are no obvious symptoms for early stage $\mathrm{CRC}$, it is usually diagnosed at advanced stages (2). Although CRC patients without metastases could be surgically cured, those in advanced stage are mainly treated with chemotherapy. Studies above indicated that chemotherapy has effective roles in preventing tumor metastasis, reducing the tumor volume and improving the clinic symptoms (3-6). However, most patients

Correspondence to: Dr Mingxia Wang, Department of Oncology, Linyi Central Hospital, 17 Jiankang Road, Linyi, Shandong 276400, P.R. China

E-mail: dfb919291953r@163.com

Key words: colorectal carcinoma, invasion, migration, miR-331-3p, neuropilin-2 eventually develop drug resistance after chemotherapy treatment. Therefore, there is a great need for a continued effort to better understand the complexity of CRC development and to identify new directions for CRC therapy.

Overwhelming evidence has shown that abnormal microRNA (miRNA/miR) expression mediated CRC development by affecting the expression of the genes which regulated tumor progression (7). miRNAs are highly conserved small non-coding RNAs, playing important functions in multiple biological processes $(8,9)$. In recent years, miRNAs have been extensively studied in tumorigenesis, including in osteosarcoma (10), glioma (11) and breast cancer (12) research. These studies indicated that miRNAs were associated with tumor pathogenesis along with the potential to develop tumor therapeutics and diagnostics. However, miR-331-3p expression patterns in human CRC and its biological mechanism still remained obscure.

Neuropilin-2 (NRP2), a member of the NRPs family, is a nontyrosine kinase transmembrane glycoprotein (13) and characterized as a receptor for the vascular semaphorin (SEMA) families and endothelial growth factor (VEGF) (14). A number of studies have notably demonstrated that the NRP2 expression is ubiquitous in various tumor cells such as lung cancer (15), cervical cancer (16) and breast cancer (17). Therefore, it is imperative to understand the specific effects of NRP2 on tumor progression. Nevertheless, the expressions and functions of NRP2 in CRC remain largely unclear. In the present study, we evaluated NRP2 expression and investigated the correlations between miR-331-3p and NRP2 in CRC.

\section{Materials and methods}

CRCtissue samples. A total of 54 pairs of human CRC tissues and matched normal tissues were collected from the Linyi Central Hospital (Linyi, China) between May 2016 and July 2018, with approval from the institutional Ethics Committee. Specimens were freshly frozen in liquid nitrogen and stored at $-80^{\circ} \mathrm{C}$ for further assays. Written informed consent from each patient was received before the samples were collected.

CRC cell culture. Human CRC cells (SW480 and HCT116) as well as normal colon cells FHC were purchased from the Chinese Academy of Sciences Cell Bank of Type Culture Collection (Shanghai, China). The cells were cultured with RPMI-1640 medium containing FBS (10\%), penicillin 
(100 U/ml) and streptomycin $(100 \mathrm{mg} / \mathrm{ml})$ in a humidified chamber $\left(37^{\circ} \mathrm{C}, 5 \% \mathrm{CO}_{2}\right)$.

Cell transfection. miR-331-3p mimics or inhibitor as well as NRP2 siRNA and the corresponding controls were purchased from Gene Pharma (Shanghai, China) and transfected into CRC cell lines by Lipofectamine ${ }^{\circledR} 2000$ (Invitrogen; Thermo Fisher Scientific, Inc.) in strict accordance with the manufacturer's instructions.

Reverse transcription (RT)-qPCR. TRIzol reagent (Invitrogen; Thermo Fisher Scientific, Inc.) was used to isolate the total RNAs from CRC cell lines or tissue samples according to the manufacturer's guidelines. Then, the extracted total RNA was used to generate the cDNA with the PrimeScript RT reagent kit (Takara Biotechnology Co., Ltd.). The temperature conditions for reverse transcription were as follows: $37^{\circ} \mathrm{C}$ for $15 \mathrm{~min}$ and $85^{\circ} \mathrm{C}$ for $5 \mathrm{sec}$. Real-time PCR assays were conducted by SYBR ${ }^{\circledR}$ Premix Ex Taq ${ }^{\mathrm{TM}}$ (Takara Biotechnology Co., Ltd.) on the ABI 7900 Sequence Detection System (Applied Biosystems; Thermo Fisher Scientific, Inc.). The miR-331-3p expression was normalized to U6 while the NRP2 was normalized to GAPDH. The primers used were as follows: For miR-331-3p: Forward, 5'-GAGCTGAAAGCACTCC CAA-3' and reverse 5'-CACACTCTTGATGTTCCAGGA-3'; for U6 forward, 5'-AGAGCCTGTGGTGTCCG-3' and reverse 5'-CATCTTCAAAGCACTTCCCT-3'; for NRP2 forward, 5'-CCCCGAACCCAACCAGAAGA-3' and reverse 5'-GAAT GCCATCCCAGATGTCCA-3'; and for GAPDH, forward 5'-GGCACTGAGAAGCGGGGCCG-3' and reverse 5'-CCC TTGTTTTTTGCTTCCCTT-3'. The thermocycling conditions were as follows: $95^{\circ} \mathrm{C}$ for $10 \mathrm{~min}$, followed by 45 cycles of denaturation at $95^{\circ} \mathrm{C}$ for $15 \mathrm{sec}$ and annealing/elongation at $60^{\circ} \mathrm{C}$ for $15 \mathrm{sec} .2^{-\Delta \Delta \mathrm{Cq}}$ method was used to determine the relative expression of the genes (18).

Immunohistochemistry (IHC). IHC was performed to detect the NRP2 expression in CRC tissues. Samples were fixed, embedded, and sliced into $4 \mu \mathrm{m}$ thick tissue sections. The sections were then dewaxed and rehydrated. For antigen retrieval, the sections were microwaved in citrate buffer for $15 \mathrm{~min}$. Then, endogenous peroxidase activity was blocked with $3 \% \mathrm{H}_{2} \mathrm{O}_{2}$. Subsequently, the sections were incubated with primary NRP2 antibody $(1: 100)$ at $4{ }^{\circ} \mathrm{C}$ overnight, and a secondary goat anti-rabbit $\operatorname{IgG}(1: 1,000)$ (both from Abcam) labeled by HRP was used for the subsequent incubation. The sections were stained with DAB solution and counterstained with haematoxylin. Images were obtained from a bright-field microscope (Olympus BX50; Olympus Corporation).

Western blot analysis. Transfected cells were lysed on ice in RIPA buffer (Thermo Fisher Scientific, Inc.) with proteinase inhibitors. Bicinchoninic acid protein (BCA) assay kit (Beyotime) was applied to measure the total protein concentrations. The protein lysates $(30 \mu \mathrm{g})$ were separated with $10 \%$ SDS-PAGE gel and then electrotransferred to PVDF which was pretreated with 5\% non-fat dry skim milk in TBST for $2 \mathrm{~h}$ at room temperature. Thereafter, the membranes were incubated with appropriate primary antibodies: anti-NRP2 (dil, 1:4,000; cat. no. ab185710); anti-GAPDH (dil, 1:1,000;
Table I. Correlation of miR-331-3p expression with the clinicopathological characteristics of the colorectal carcinoma patients.

\begin{tabular}{|c|c|c|c|c|}
\hline \multirow[b]{2}{*}{$\begin{array}{l}\text { Clinicopathological } \\
\text { features }\end{array}$} & \multirow[b]{2}{*}{$\begin{array}{l}\text { Cases } \\
(\mathrm{n}=54)\end{array}$} & \multicolumn{2}{|c|}{$\begin{array}{c}\operatorname{miR}-331-3 \mathrm{p}^{\mathrm{a}} \\
\text { expression }\end{array}$} & \multirow[b]{2}{*}{ P-value } \\
\hline & & $\begin{array}{l}\text { High } \\
(n=18)\end{array}$ & $\begin{array}{c}\text { Low } \\
(n=36)\end{array}$ & \\
\hline Age (years) & & & & 0.563 \\
\hline$>60$ & 26 & 10 & 16 & \\
\hline$\leq 60$ & 28 & 8 & 20 & \\
\hline Sex & & & & 0.471 \\
\hline Male & 30 & 12 & 18 & \\
\hline Female & 24 & 6 & 18 & \\
\hline Tumor size $(\mathrm{cm})$ & & & & 0.312 \\
\hline$\geq 5.0$ & 27 & 7 & 20 & \\
\hline$<5.0$ & 27 & 11 & 16 & \\
\hline TNM stage & & & & $0.015^{\mathrm{b}}$ \\
\hline I-II & 21 & 15 & 6 & \\
\hline III & 33 & 3 & 30 & \\
\hline $\begin{array}{l}\text { Lymph node } \\
\text { metastasis }\end{array}$ & & & & $0.006^{\mathrm{b}}$ \\
\hline Yes & 31 & 5 & 26 & \\
\hline No & 23 & 13 & 10 & \\
\hline \multicolumn{5}{|l|}{ Location } \\
\hline Colon & 27 & 12 & 15 & 0.316 \\
\hline Rectum & 27 & 6 & 21 & \\
\hline Distant metastasis & & & & 0.072 \\
\hline Yes & 28 & 9 & 19 & \\
\hline No & 26 & 9 & 17 & \\
\hline
\end{tabular}

${ }^{a}$ The mean expression level of miR-331-3p was used as the cut-off; ${ }^{\mathrm{b}}$ statistically significant. TNM, tumor-node-metastasis.

cat. no. ab181603) E-cadherin (dil, 1:2,000; cat. no. ab15148), N-cadherin (dil, 1:2,000; cat. no. ab18203), Vimentin (dil, 1:1,000; cat. no. ab137321) (all from Abcam) overnight at $4^{\circ} \mathrm{C}$. The membrane was then incubated with anti-rabbit IgG (dil, 1:5,000; cat. no. ab191866; Abcam) at room temperature for $2 \mathrm{~h}$. The protein bands were detected by chemiluminescent detection system (Beyotime). GAPDH was the internal reference.

Transwell assays. Cell invasion and migration assays were performed by Transwell chambers (Coring Costar) with membrane pore size of $8.0 \mu \mathrm{m}$. After treated with miR-331-3p mimics, inhibitor or NRP2 siRNA, CRC cell lines were seeded into the top chamber. For invasion and migration assays, Transwell chamber was pretreated with or without Matrigel (BD Biosciences) respectively. The top chamber was added with serum-free medium when the medium containing $10 \%$ FBS was added into the bottom chambers. After incubation for $48 \mathrm{~h}$ at $37^{\circ} \mathrm{C}$, the cells that remained on the top surface were removed with cotton swabs. At the same 

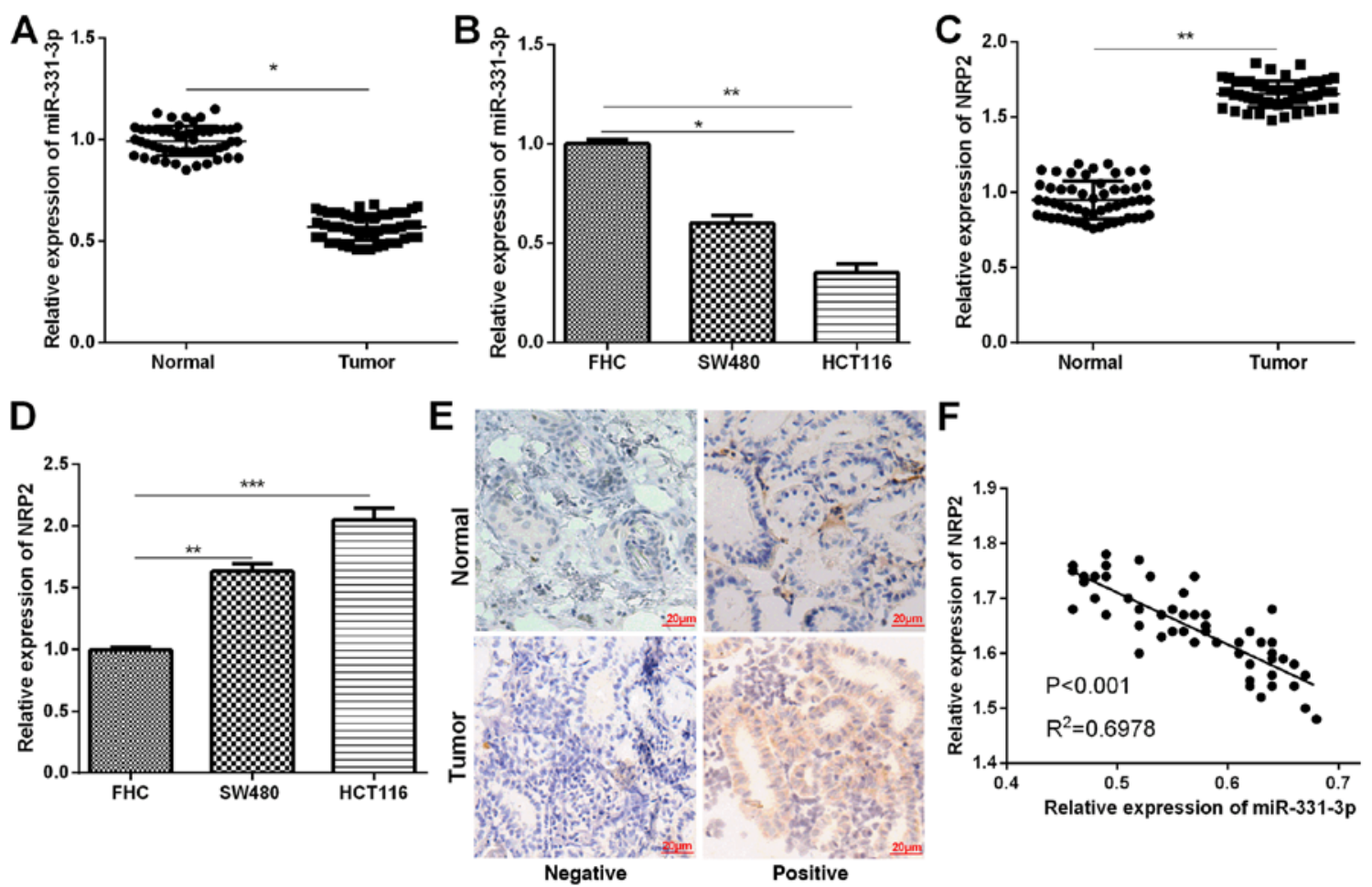

Figure 1. Expressions analysis of miR-331-3p and NRP2 in CRC tissue samples and cells. (A and B) The relative expression of miR-331-3p in CRC tissue samples $(\mathrm{n}=54)$ and cell lines were measured by RT-qPCR. (C and D) The relative expression of NRP2 in CRC tissues and cell lines were detected by RT-qPCR . (E) Expression of NRP2 in CRC tissues was measured by IHC. (F) Correlation between expression of miR-331-3p and NRP2. ${ }^{*} \mathrm{P}<0.05,{ }^{* *} \mathrm{P}<0.01,{ }^{* * *} \mathrm{P}<0.001$. The data are from at least 3 independent experiments. CRC, colorectal carcinoma; NRP2, neuropilin-2; IHC, immunohistochemistry.

time, those that adhered to the bottom surface were fixed and stained respectively using formaldehyde $(4 \%)$ and crystal violet $(0.1 \%)$ for detecting the images using a microscope (Olympus Corporation). The values for invasion or migration were obtained by counting 3 randomly selected fields per membrane and represented the average of 3 independent experiments.

In silico analysis and luciferase reporter assay. TargetScan database (http://www.targetscan.org/vert_72/) was utilized to scan for the potential target gene of miR-331-3p that may participate in CRC (19).

The amplified NRP2-3'-UTR-WT and corresponding NRP2-3'-UTR-MUT were respectively inserted into pGL3 luciferase vectors (Promega). CRC cells were cotransfected with miR-331-3p mimics and luciferase reporter vectors of the wild-type or mutant-type 3'-UTR of NRP2 by Lipofectamine $^{\mathrm{TM}} 2000$ (Invitrogen; Thermo Fisher Scientific, Inc.). Subsequently, the Dual luciferase reporter assay kit (Promega) was used to detect the relative luciferase activities $48 \mathrm{~h}$ after the transfections.

Statistical analysis. The above assays were conducted at least three times. SPSS 17.0 (SPSS Inc.) was used to perform the statistical analysis with Student's t-test or one-way ANOVA test followed by post hoc test. Data are indicated as means \pm SD. Correlation between expression levels of miR-331-3p and NRP2 was estimated using the Pearson's correlation method. The differences were identified as statistically significant at $\mathrm{P}<0.05$.

\section{Results}

miR-331-3p is downregulated and NRP2 is upregulated in $C R C$. To determine whether miR-331-3p was involved in CRC carcinogenesis, its expression in 54 pairs of CRC tissue samples and two cell lines was detected by RT-qPCR. The results indicated that, when compared to the matched normal tissue samples, the miR-331-3p expression in CRC tissue samples was significantly decreased (Fig. 1A). Similarly, RT-qPCR results also indicated that miR-331-3p expression in CRC cells was significantly lower than that in normal colonic cells (Fig. 1B). Furthermore, NRP2 expression levels in CRC tissues and cells were measured. RT-qPCR analysis demonstrated significantly higher mRNA levels of NRP2 in both CRC tissues and cells compared to the corresponding controls (Fig. 1C and D). In addition, we analyzed the correlation between the expression of NRP2 and miR-331-3p in CRC tissues to better understand their functions in CRC progression. The results demonstrated that the miR-331-3p expression had a negative correlation with the expression of NRP2 in CRC tissues (Fig. 1E). In addition, all the enrolled CRC patients were assigned into high or low miR-331-3p expression groups based on the mean miR-331-3p level. Clinicopathologic analysis demonstrated that CRC patients with low miR-331-3p expression presented malignant clinicopathological features (Table I).

miR-331-3p inhibits CRC cell invasion and migration. To further understand the effects of miR-331-3p on CRC progression, SW480 and HCT116 cells were trasnfected with miR-331-3p mimics or inhibitor to overexpress or inhibit 
A
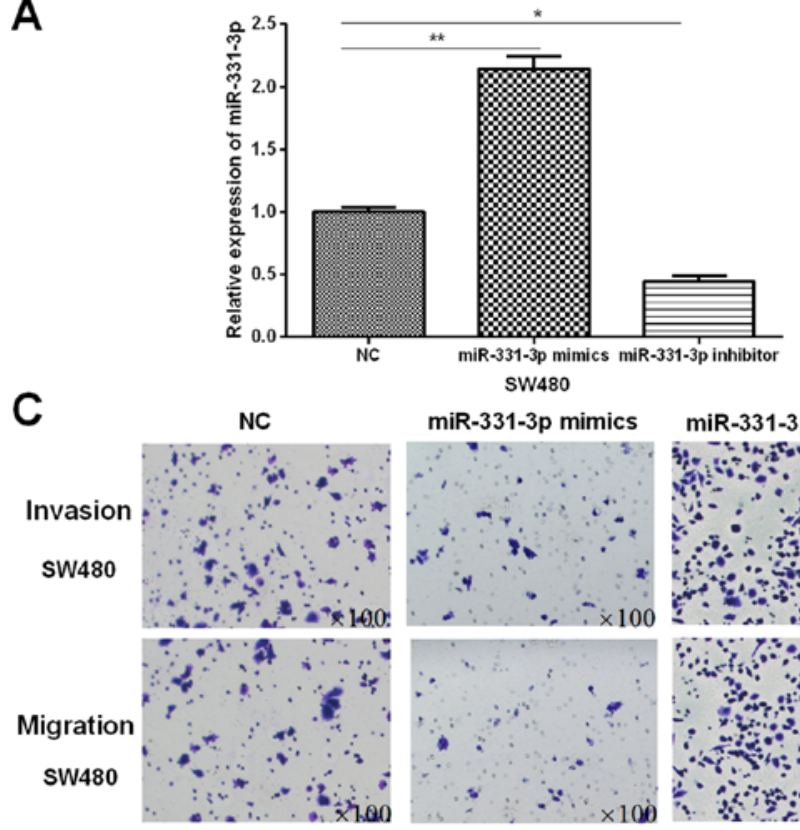

D

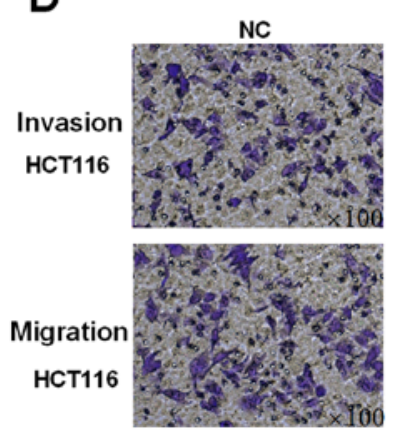

miR-331-3p mimics
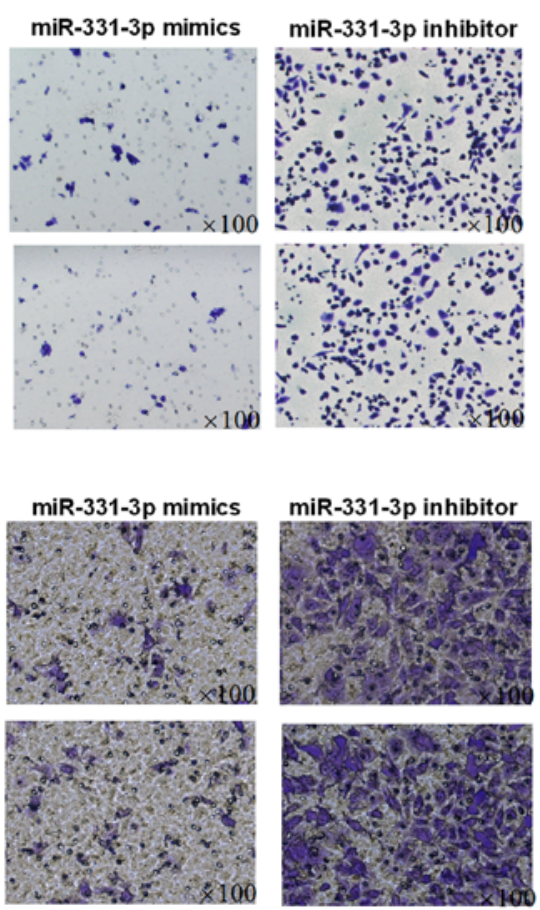

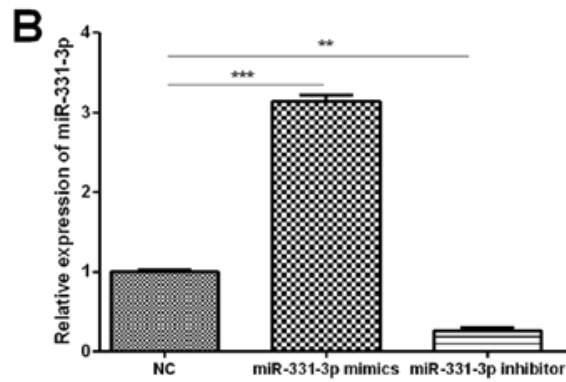

HCT116

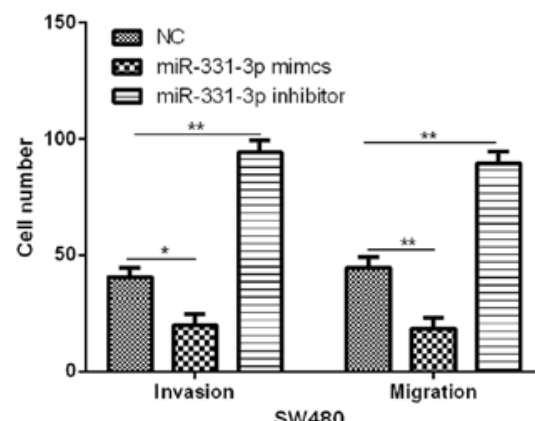

SW480

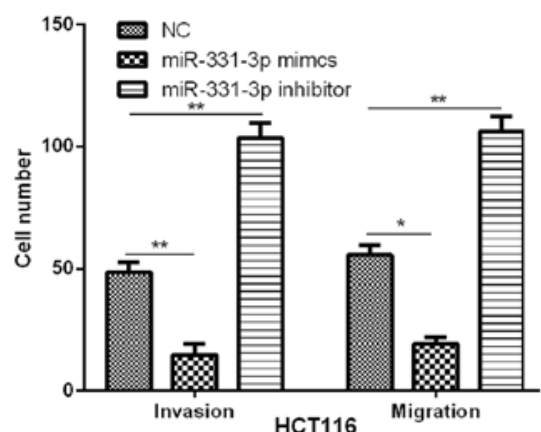

Figure 2. miR-331-3p inhibits cell invasion and migration in CRC cells. (A and B) The miR-331-3p expression in transfected CRC cell lines was evaluated using RT-qPCR. (C) The functions of miR-331-3p in SW480 invasion or migration were analyzed by Transwell assays. (D) Transwell assays were carried out to detect cell invasion and migration in transfected HCT116 cells. ${ }^{*} \mathrm{P}<0.05,{ }^{* *} \mathrm{P}<0.01,{ }^{* * *} \mathrm{P}<0.001$. CRC, colorectal carcinoma.

miR-331-3p expression. RT-qPCR analysis was performed to confirm the successful miR-331-3p overexpression or downregulation in SW480 or HCT116 cells (Fig. 2A and B). Subsequently, we explored the functions of miR-331-3p in SW480 and HCT116 cell invasion and migration through performing Transwell assays. Fig. 2C shows that overexpression of miR-331-3p could markedly repress the invasion and migration capacities of SW480 cells when decreased expression of miR-331-3p enhanced the SW480 invasion and migration. Additionally, similar functions of miR-331-3p in HCT116 cell invasion and migration were confirmed by Transwell assays (Fig. 2D). Results suggested that miR-331-3p was able to inhibit CRC cell invasion and migration.

miR-331-3p upregulation suppresses CRC cell EMT. It was reported that EMT is regarded as a crucial representations in cancer metastasis and invasion. Thus, to address molecular mechanism of miR-331-3p-induced anti-metastatic effect on CRC cells, western blot analysis was performed to detect the protein levels involved in EMT occurrence. It was demonstrated that in SW480 cells, the expression levels of E-cadherin were significantly increased while the expression levels of $\mathrm{N}$-cadherin and Vimentin were significantly decreased by
miR-331-3p mimics (Fig. 3A). On the other hand, miR-331-3p inhibitor in SW480 cells had the opposite functions in EMT-related proteins (Fig. 3B). Moreover, we examined the protein expression in HCT116 cells, and a similar influence of miR-331-3p on the expression of proteins which were closely related to EMT was identified (Fig. 3C and D).

miR-331-3p interacts with NRP2 in CRC cells by directly binding to the NRP2 3'-UTR. The correlation was investigated between NRP2 and miR-331-3p to fully understand the mechanisms of miR-331-3p in regulating CRC. Based on Targetscan, miR-331-3p was predicted to bind to NRP2 3'-UTR (Fig. 4A), suggesting that NRP2 was a potential target for miR-331-3p. Then, to confirm whether NRP2 was directly targeted by miR-331-3p, we performed dual-luciferase reporter assays. The luciferase reporter vectors which contained NRP2 3'-UTR-WT or NRP2 3'-UTR-MUT were constructed and cotransfected into CRC cells with miR-331-3p mimics. The relative luciferase activities of the reporter containing the NRP2 3'-UTR-WT were significantly reduced by miR-331-3p mimics; however, the luciferase activity of NRP2 3'-UTR-MUT was not notably affected by miR-331-3p mimics (Fig. 4B). RT-qPCR and western blot analyses of the NRP2 expression 

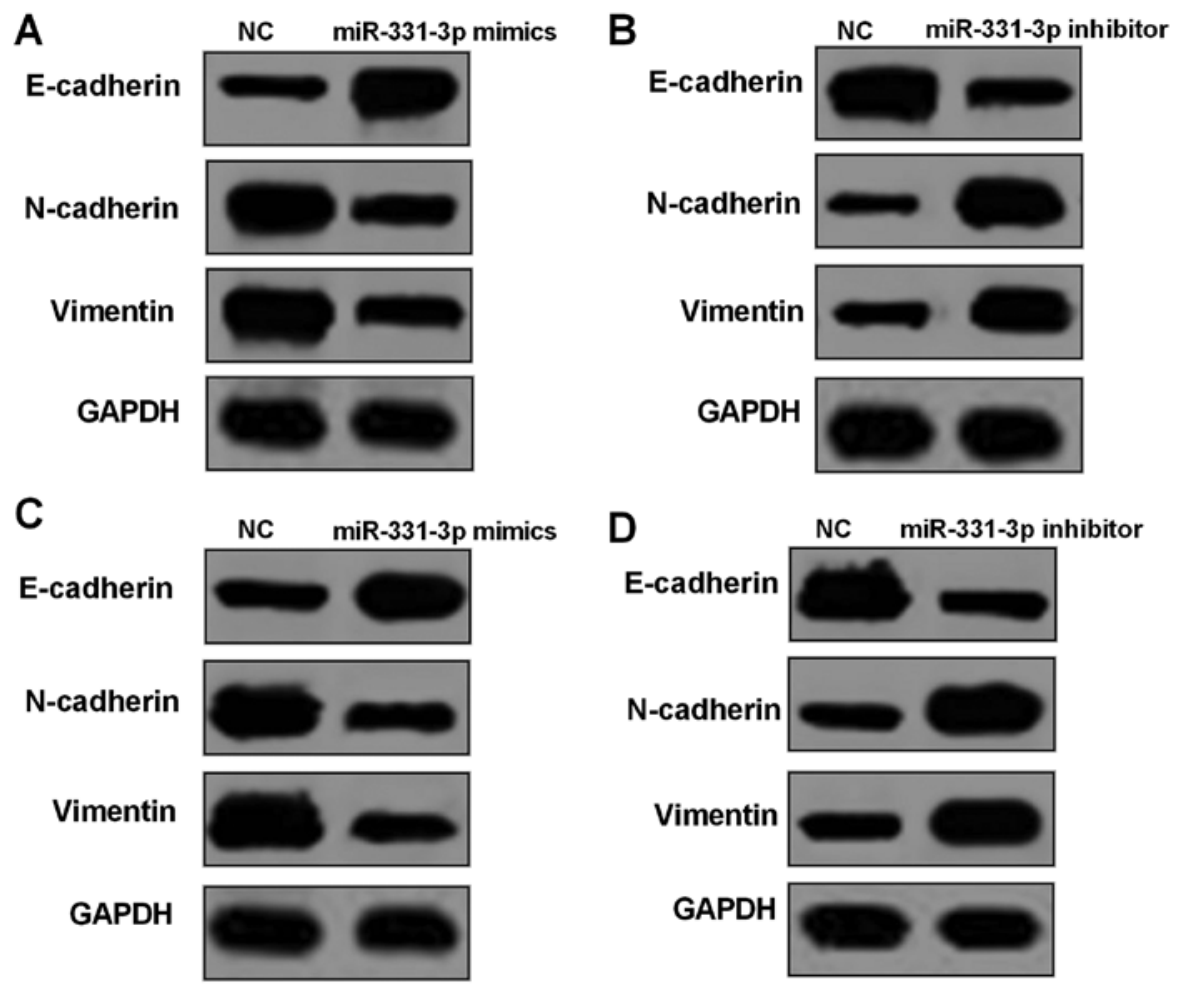

Figure 3. miR-331-3p inhibits CRC cell EMT. (A and B) Western blot analysis was performed to detect the expression levels of EMT-related proteins in transfected SW480 cells. (C and D) Expression levels of EMT-related proteins in transfected HCT116 cells.

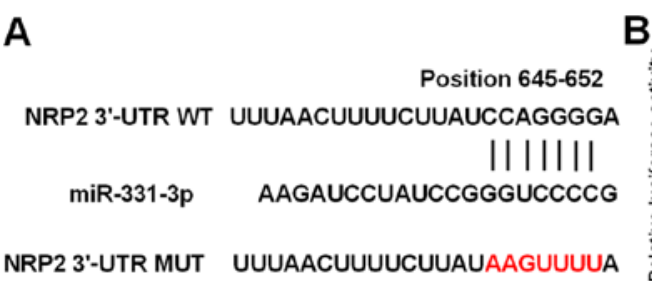

C

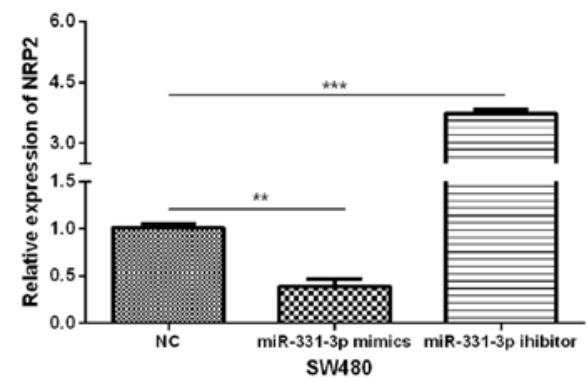

D

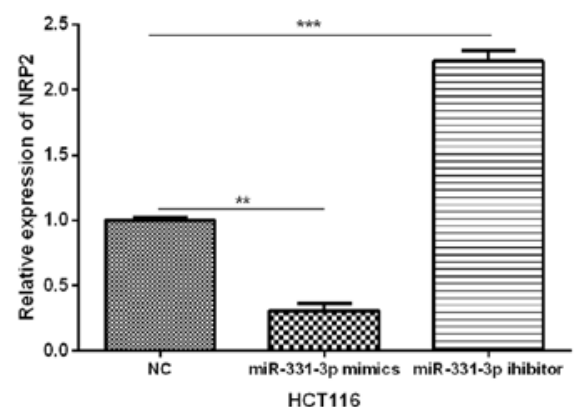

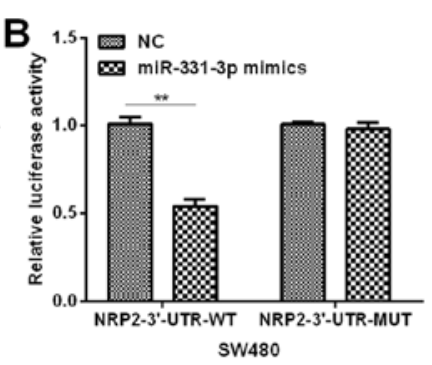
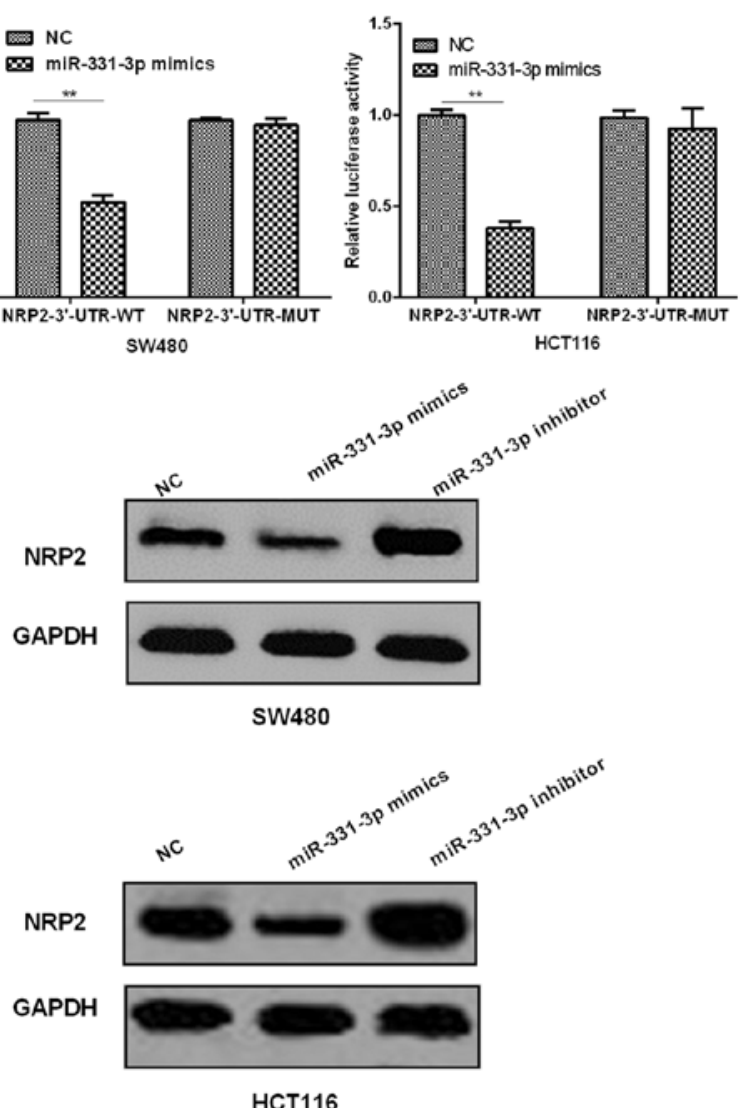

HCT116

Figure 4. miR-331-3p deregulates NRP2 expression via binding to NRP2 3'-UTR directly. (A) The binding sequences of miR-331-3p in NRP2 3'-UTR. (B) The luciferase reporter gene assays were carried out to measure the fluorescence activities of NRP2 3'-UTR in CRC cells which were co-transfected with NRP2-3'-UTR-WT or NRP2-3'-UTR-MUT and miR-331-3p mimics, respectively. (C and D) RT-qPCR and western blot results of NRP2 expression levels in CRC cells with different transfections ${ }^{* * *} \mathrm{P}<0.01,{ }^{* * *} \mathrm{P}<0.001$. CRC, colorectal carcinoma; NRP2, neuropilin-2. 
A

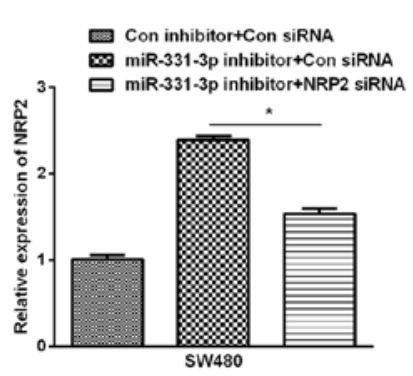

C

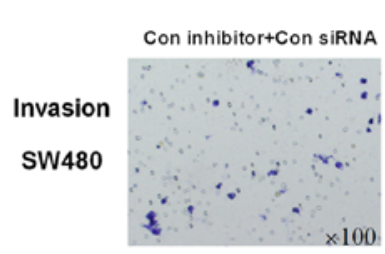

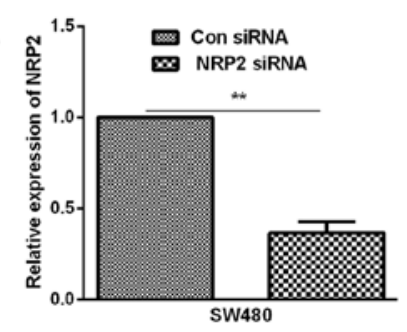

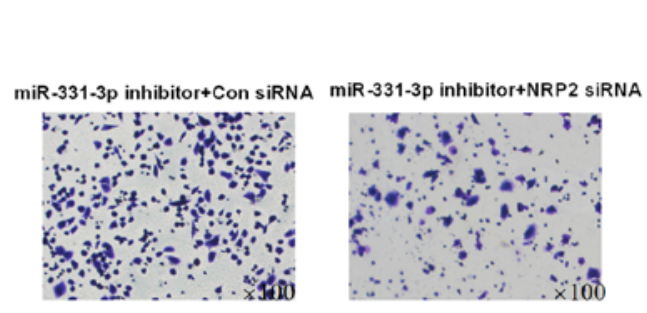

B
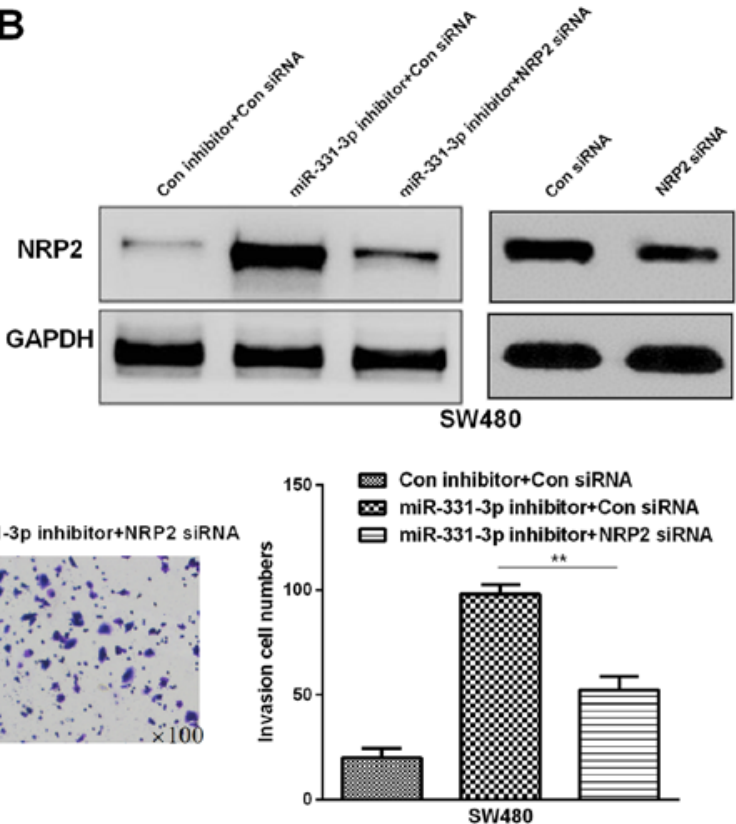

D
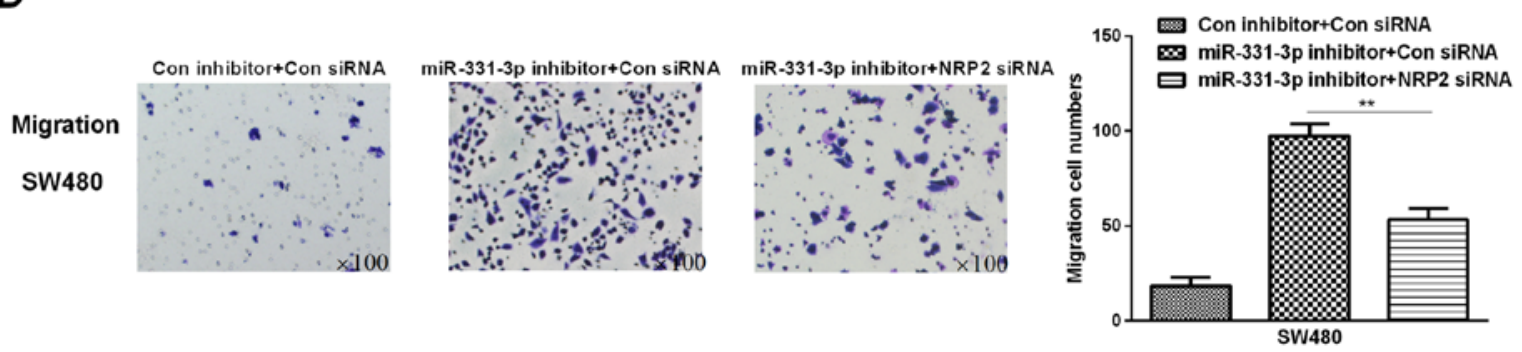

Figure 5. Knockdown of NRP2 abrogates the function mediated by miR-331-3p inhibitor in CRC cell invasion and migration. (A and B) NRP2 mRNA or protein expression levels were measured using RT-qPCR or western blots in CRC cells with different transfections. (C and D) Transwell assays were conducted to observe invasion and migration capacity in $\mathrm{CRC}$ cells cotransfected with NRP2 siRNA and miR-331-3p inhibitor. ${ }^{*} \mathrm{P}<0.05 ;{ }^{* *} \mathrm{P}<0.01$. CRC, colorectal carcinoma; NRP2, neuropilin-2.

demonstrated that NRP2 expression levels in CRC cells were significantly decreased by miR-331-3p mimics in contrast to the controls, whereas NRP2 expressions in cells with transfection of miR-331-3p inhibitor demonstrated notably increased NRP2 levels compared with the NC (Fig. 4C and D).

Silencing of NRP2 partially reverses the miR-331-3p inhibitor-mediated functions in promoting SW480 cell invasion and migration. To elucidate whether miR-331-3p exerted anti-CRC functions through regulating NRP2, NRP2 siRNA and miR-331-3p inhibitor were co-transfected into CRC cell lines. NRP2 siRNA was transfected into CRC cells to knock down NRP2, RT-qPCR and western blot results showed that, transfection with NRP2 siRNA resulted in marked downregulation of NRP2 expression in CRC cells (Fig. 5A and B). Moreover, similar results were also identified in CRC cells transfected with NRP2 siRNA and miR-331-3p inhibitor (Fig. 5A and B). Subsequently, the Transwell assays were carried out to determine the functions of NRP2 siRNA in SW480 cell migration and invasion. Results demonstrated that the invasion and migration abilities of SW480 cell lines cotransfected with miR-331-3p inhibitor and NRP2 siRNA were markedly suppressed compared to that of the only miR-331-3p downregulated SW480 cell lines (Fig. 5D and E).
The findings suggested that deletion of NRP2 markedly reversed miR-331-3p inhibitor-mediated promotion of cell invasion and migration in SW480 cell lines.

\section{Discussion}

$\mathrm{CRC}$ is a critical challenge both for public health and clinical practice. In recent decades, although the life expectancy of CRC patients has been improved due to the advances in CRC screening and therapy (20), CRC still remains a leading health problem worldwide. Thus, more attention should been given to the specific mechanisms of the CRC initiation and development. Growing evidence has indicated that miRNAs play important functions in human CRC development (21). Moreover, miRNAs have been determined to play a crucial role in regulating gene expression, and in other relevant processes, such as invasion and metastasis (22).

miR-331-3p has been identified as a tumor-associated miRNA. As an independent prognostic factor, miR-331-3p was reported to modulate tumor progression. Epis et al (23) found that miR-331-3p inhibited prostate cancer progression with Aurora Kinase inhibitor II cotreatment; Chen et al(24) reported that in hepatocellular carcinoma patients, serum miR-331-3p and miR-182 functioned as therapic biomarkers; 
Cao et al (25) verified that miR-331-3p suppressed VHL expression in HCC. Given that miRNAs are widely known as tumor regulators, we provide further evidence in this study that miR-331-3p plays important roles in human CRC. miR-331-3p was identified as the downregulated miRNA in CRC by RT-qPCR. Moreover, we found that decreased miR-331-3p was associated with the aggressive clinicopathological features of CRC patients. Over-expression of miR-331-3p was able to inhibit CRC cell invasion and migration by targeting NRP2 and regulating EMT. Collectively, the findings of this research revealed that miR-331-3p played anti-tumor roles in CRC.

Neuropilins (NRPs) are type I transmembrane receptors that form heterodimeric complexes with two key classes of signaling transmembrane receptors: Plexins and vascular endothelial growth factor receptors (VEGFRs) (26). There are two main NRP receptors (NRP1 and NRP2), with multiple extracellular and transmembrane isoforms observed for each in vivo (27). NRPs are thought primarily to modulate the affinity and specificity of extracellular ligand binding upon co-receptor complex formation. Plexin-NRP co-receptor complexes bind semaphorins (Semas), which are a large class of extracellular, dimeric ligands that act as either attractive or repulsive cues during cell migration in a diverse array of processes (28). VEGFR-NRP co-receptor complexes bind vascular endothelial growth factor (VEGF), which plays a major role in the induction of endothelial cell proliferation and increase of the vascular endothelium permeability $(29,30)$. NRP is now considered a candidate specific receptor for VEGF (31). Given the diversity of biological processes in which Sema and VEGF modulate cell migration, dysregulation of NRP-dependent signaling has been linked to a variety of cancers. The role of NRPs as co-receptors of Semas and VEGF in tumor angiogenesis and metastases is the basis for current trials. Various research has reported the effects and mechanisms of NRP2 on tumor progression. Fung et al (32) indicated that NRP2 promoted oesophageal squamous cell carcinoma metastasis and tumorigenicity; Dallas et al (33) further demonstrated that NRP2 regulated pancreatic adenocarcinoma angiogenesis and growth; Moriarty et al (34) found that NRP2 promoted melanoma progression and growth. To our knowledge, there is no previous report on research investigating the association between NRP2 and miR-331-3p in CRC. The current study provided preliminary strong evidence that NRP2 was directly targeted by miR-331-3p and implicated in CRC invasion and migration. The data also revealed that knockdown of NRP2 reversed the functions of miR-331-3p inhibitor in cell invasion and migration of CRC cells. These results suggest that miR-331-3p exerted cancer suppressive roles in CRC via targeting NRP2.

In conclusion, miR-331-3p was downregulated in CRC, which indicates poor outcomes of CRC patients. miR-331-3p overexpression suppressed migration and invasion through regulating NRP2 and EMT. In addition, the suppression function of miR-331-3p in invasion and migration of CRC cells was partially mediated by direct deregulation of NRP2. Thus, the findings in the current study may help to better determine the mechanisms of miR-331-3p and NRP2 implicated in CRC progression, and to discover sensitive prognostic and therapeutic biomarkers for CRC.

\section{Acknowledgements}

Not applicable.

\section{Funding}

This study was supported by Shandong Traditional Chinese Medicine Science and Technology Development Plan (2017-474).

\section{Availability of data and materials}

The datasets used and/or analyzed during the current study are available from the corresponding author on reasonable request.

\section{Authors' contributions}

$\mathrm{HZ}$ as the first author contributed significantly to statistics analysis and manuscript preparation. RW wrote the manuscript and helped perform the statistics analysis with constructive discussions. MW as the corresponding author contributed to the conception of the study and provided clinical data of the patients as well as crucial experiment materials. All authors read and approved the final manuscript.

\section{Ethics approval and consent to participate}

The study was approved by the Ethics Committee of Linyi Central Hospital (Linyi, China). Written informed consent from each patient was received before the samples were collected.

\section{Patient consent for publication}

Not applicable.

\section{Competing interests}

The authors declare that they have no competing interests.

\section{References}

1. Bae JM, Kim JH, Kwak Y, Lee DW, Cha Y, Wen X, Lee TH, Cho NY, Jeong SY, Park KJ, et al: Distinct clinical outcomes of two CIMP-positive colorectal cancer subtypes based on a revised CIMP classification system. Br J Cancer 116: 1012-1020, 2017.

2. Hadjipetrou A, Anyfantakis D, Galanakis CG, Kastanakis M and Kastanakis S: Colorectal cancer, screening and primary care: A mini literature review. World J Gastroenterol 23: 6049-6058, 2017.

3. Haraldsdottir S, Einarsdottir HM, Smaradottir A, Gunnlaugsson A and Halfdanarson TR: Colorectal cancerreview. Laeknabladid 100: 75-82, 2014 (In Icelandic).

4. Lieberman D, Ladabaum U, Cruz-Correa M, Ginsburg C, Inadomi JM, Kim LS, Giardiello FM and Wender RC: Screening for colorectal cancer and evolving issues for physicians and patients: A Review. JAMA 316: 2135-2145, 2016.

5. Liu W, Sun Y, Zhang L and Xing BC: Negative surgical margin improved long-term survival of colorectal cancer liver metastases after hepatic resection: A systematic review and meta-analysis. Int J Colorectal Dis 30: 1365-1373, 2015.

6. Massat NJ, Moss SM, Halloran SP and Duffy SW: Screening and primary prevention of colorectal cancer: A review of sex-specific and site-specific differences. J Med Screen 20: 125-148, 2013

7. Bonfrate L, Altomare DF, Di Lena M, Travaglio E, Rotelli MT, De Luca A and Portincasa P: MicroRNA in colorectal cancer: New perspectives for diagnosis, prognosis and treatment. J Gastrointestin Liver Dis 22: 311-320, 2013. 
8. Karatas OF, Suer I, Yuceturk B, Yilmaz M, Hajiyev Y, Creighton CJ, Ittmann M and Ozen M: The role of miR-145 in stem cell characteristics of human laryngeal squamous cell carcinoma Hep-2 cells. Tumour Biol 37: 4183-4192, 2016.

9. Xu L, Li Q, Xu D, Wang Q, An Y, Du Q, Zhang J, Zhu Y and Miao Y: hsa-miR-141 downregulates TM4SF1 to inhibit pancreatic cancer cell invasion and migration. Int J Oncol 44: 459-466, 2014

10. Wang G, Shen N, Cheng L, Lin J and Li K: Downregulation of miR-22 acts as an unfavorable prognostic biomarker in osteosarcoma. Tumour Biol 36: 7891-7895, 2015.

11. Bai QL, Hu CW, Wang XR, Shang JX and Yin GF: miR-616 promotes proliferation and inhibits apoptosis in glioma cells by suppressing expression of SOX7 via the Wnt signaling pathway. Eur Rev Med Pharmacol Sci 21: 5630-5637, 2017.

12. Wang Z, Liao H, Deng Z, Yang P, Du N, Zhanng Y and Ren H: miRNA-205 affects infiltration and metastasis of breast cancer. Biochem Biophys Res Commun 441: 139-143, 2013.

13. Staton CA, Kumar I, Reed MW and Brown NJ: Neuropilins in physiological and pathological angiogenesis. J Pathol 212: 237-248, 2007.

14. Pellet-Many C, Frankel P, Jia $\mathrm{H}$ and Zachary I: Neuropilins: Structure, function and role in disease. Biochem J 411: 211-226, 2008.

15. Drabkin HA, Starkova J and Gemmill RM: A triad of NRP2, DLX and p53 proteins in lung cancer metastasis. Oncotarget 8: 96464-96465, 2017

16. Fujii T, Shimada K, Asano A, Tatsumi Y, Yamaguchi N, Yamazaki $\mathrm{M}$ and Konishi N: MicroRNA-331-3p suppresses cervical cancer cell proliferation and E6/E7 expression by targeting NRP2. Int J Mol Sci 17: 17, 2016.

17. Yasuoka H, Kodama R, Tsujimoto M, Yoshidome $K$, Akamatsu $H$ Nakahara M, Inagaki M, Sanke T and Nakamura Y: Neuropilin-2 expression in breast cancer: Correlation with lymph node metastasis, poor prognosis, and regulation of CXCR4 expression. BMC Cancer 9: 220, 2009.

18. Livak KJ and Schmittgen TD: Analysis of relative gene expression data using real-time quantitative PCR and the 2 (-Delta DeltaC(T)) method. Methods 25: 402-408, 2001

19. Dweep H, Sticht C, Pandey P and Gretz N: miRWalk - database: Prediction of possible miRNA binding sites by 'walking' the genes of three genomes. J Biomed Inform 44: 839-847, 2011.

20. Burstein HJ and Schwartz RS: Molecular origins of cancer. N Engl J Med 358: 527, 2008.

21. El Sharawy A, Röder C, Becker T, Habermann JK, Schreiber S, Rosenstiel $\mathrm{P}$ and Kalthoff $\mathrm{H}$ : Concentration of circulating miRNA-containing particles in serum enhances miRNA detection and reflects CRC tissue-related deregulations. Oncotarget 7: 75353-75365, 2016.

22. Suzuki HI, Katsura A, Matsuyama $\mathrm{H}$ and Miyazono K: MicroRNA regulons in tumor microenvironment. Oncogene 34 3085-3094, 2015.
23. Epis MR, Giles KM, Beveridge DJ, Richardson KL, Candy PA Stuart LM, Bentel J, Cohen RJ and Leedman PJ: miR-331-3p and Aurora kinase inhibitor II co-treatment suppresses prostate cancer tumorigenesis and progression. Oncotarget 8: 55116-55134, 2017.

24. Chen L, Chu F, Cao Y, Shao J and Wang F: Serum miR-182 and miR-331-3p as diagnostic and prognostic markers in patients with hepatocellular carcinoma. Tumour Biol 36: 7439-7447, 2015.

25. Cao Y, Zhang J, Xiong D, Wang D, Wu T, Huang A and Tang H Hsa-miR-331-3p inhibits VHL expression by directly targeting its mRNA 3'-UTR in HCC cell lines. Acta Biochim Pol 62: 77-82, 2015.

26. Zachary IC, Frankel P, Evans IM and Pellet-Many C: The role of neuropilins in cell signalling. Biochem Soc Trans 37: 1171-1178, 2009.

27. Rossignol M, Gagnon ML and Klagsbrun M: Genomic organization of human neuropilin-1 and neuropilin-2 genes: Identification and distribution of splice variants and soluble isoforms. Genomics 70: 211-222, 2000.

28. Niland S and Eble JA: Neuropilins in the context of tumor vasculature. Int J Mol Sci 20: 20, 2019.

29. Favier B, Alam A, Barron P, Bonnin J, Laboudie P, Fons P, Mandron M, Herault JP, Neufeld G, Savi P, et al: Neuropilin-2 interacts with VEGFR-2 and VEGFR-3 and promotes human endothelial cell survival and migration. Blood 108: 1243-1250, 2006.

30. Ferrara N, Gerber HP and LeCouter J: The biology of VEGF and its receptors. Nat Med 9: 669-676, 2003.

31. Caunt M, Mak J, Liang WC, Stawicki S, Pan Q, Tong RK, Kowalski J, Ho C, Reslan HB, Ross J, et al: Blocking neuropilin-2 function inhibits tumor cell metastasis. Cancer Cell 13: 331-342, 2008.

32. Fung TM, Ng KY, Tong M, Chen JN, Chai S, Chan KT, Law S, Lee NP, Choi MY, Li B, et al: Neuropilin-2 promotes tumourigenicity and metastasis in oesophageal squamous cell carcinoma through ERK-MAPK-ETV4-MMP-E-cadherin deregulation. J Pathol 239: 309-319, 2016.

33. Dallas NA, Gray MJ, Xia L, Fan F, van Buren G II, Gaur P, Samuel S, Lim SJ, Arumugam T, Ramachandran V, et al: Neuropilin-2-mediated tumor growth and angiogenesis in pancreatic adenocarcinoma. Clin Cancer Res 14: 8052-8060, 2008.

34. Moriarty WF, Kim E, Gerber SA, Hammers H and Alani RM: Neuropilin-2 promotes melanoma growth and progression in vivo. Melanoma Res 26: 321-328, 2016.

This work is licensed under a Creative Common Attribution-NonCommercial-NoDerivatives 4.0 International (CC BY-NC-ND 4.0) License. 